\title{
COMMISSIONING AND FIRST OPERATION OF THE ANTIPROTON DECELERATOR (AD)
}

\author{
P. Belochitskii, J. Bosser, J. Buttkus, C. Carli, F. Caspers, V. Chohan, D. Cornuet, T. Eriksson, \\ A. Findlay, M. Giovannozzi, B. Holzer, R. Maccaferri, N. Madsen, M. Marchesotti, S. Maury, \\ D. Mohl, S. Pasinelli, F. Pedersen, L. Soby, J. Tan, G. Tranquille, CERN, Geneva, Switzerland.
}

\begin{abstract}
Ab stract
The Antiproton Decelerator (AD) is a simplified source of antiprotons which provides low energy antiprotons for experiments, replacing four machines:

AC (Antiproton Collector), AA (Antiproton Accumulator), PS and LEAR (Low Energy Antiproton Ring), shutdown in 1996. The former AC was modified to include deceleration and electron cooling. The $\mathrm{AD}$ started operation in July 2000 and has since delivered cooled beam at $100 \mathrm{MeV} / \mathrm{c}$ (kinetic energy of $5.3 \mathrm{MeV}$ ) to 3 experiments (ASACUSA, ATHENA and ATRAP) for $1500 \mathrm{~h}$. The flux (up to $2.5 \times 10^{5} \mathrm{pbars} / \mathrm{s}$ delivered in short pulses of $330 \mathrm{~ns}$ every $110 \mathrm{~s}$ ) and the quality of the ejected beam are not far from the design specifications. A linear RF Quadrupole Decelerator (RFQD) was commissioned in November 2000 to post-decelerate the beam for ASACUSA from $5.3 \mathrm{MeV}$ to about $15 \mathrm{keV}$. Problems encountered in converting the fixed energy AC into a decelerating machine will be outlined, and the present status of the $\mathrm{AD}$, including the performance of the cooling systems and the special diagnostics to cope with beams of less than $10^{7}$ pbars, will be reviewed. Possible future developments will be sketched.
\end{abstract}

\section{INTRODUCTION}

The new low-energy antiproton facility at CERN was successfully commissioned, starting in fall 1998 (Fig. 1) [1]. A first four-month particle physics run took place from July to October 2000 followed by a second run, which started after the shutdown of the CERN machines at the end of April 2001. Beams at 5.3 MeV (100 MeV/c) with characteristics summarised in table 1 are more or less routinely delivered to the three experiments (ATHENA, ATRAP and ASACUSA).

The first two experiments which are supposed to produce antihydrogen, use degrader foils for the last deceleration from $5.3 \mathrm{MeV}$ to near rest, while ASACUSA (Atomic Spectroscopy and Collisions Using Slow Antiprotons) uses a decelerating RFQ (RFQD). This RFQD [2] was installed in the experimental area in October 2000 and has been successfully commissioned. The AD performance, approaching the design expectation (except for a factor of 2 in the cycle time), is consistently obtained when all systems work "correctly". However, there are still periods with reduced performance or complete breakdown, due to difficulties with various hardware and software components. In this status report, some conclusions are draw from the commissioning and first year of operation, with prospects for future improvements.

\section{BASIC DESCRIPTION}

The AD is a simplified scheme to provide low-energy antiprotons. It consists of just one ring, the former 3.5 $\mathrm{GeV} / \mathrm{c}$ Antiproton Collector (AC), which has been modified to include deceleration down to $0.1 \mathrm{GeV} / \mathrm{c}$ [3]. The beam from the production target is injected, rfdebunched ('bunch rotation'), and stochastically cooled at $3.5 \mathrm{GeV} / \mathrm{c}$ (Fig. 2). This is followed by rf-deceleration and further cooling on three intermediate plateaux: 2 $\mathrm{GeV} / \mathrm{c}$ (stochastic cooling) and 0.3 and $0.1 \mathrm{GeV} / \mathrm{c}$ (electron cooling). Prior to fast extraction, the beam is recaptured by a $160 \mathrm{kHz}(\mathrm{h}=1)$ rf-system and transformed into a single bunch of $300 \mathrm{~ns}$ length (bunching factor 1/20). The overall gain in 6-dimensional phase space density is more than $10^{11}$.

Almost $90 \%$ of the equipment has been recuperated from the AC (target area, injection/ejection lines, hall, magnets, power supplies, rf, stochastic cooling, diagnostics...). Further components come from LEAR (electron cooling, additional supplies, and beam line equipment) and from the AA. In this way a $100 \mathrm{MCHF}$

Table 1: AD performance at $100 \mathrm{MeV} / \mathrm{c}$

\begin{tabular}{|c|c|c|c|}
\hline Extracted beam characteristic & Operational & Design aim & \\
\hline Momentum & 100 & 100 & $\mathrm{MeV} / \mathrm{c}$ \\
\hline Intensity & $2 \times 10^{7}$ & $1.2 \times 10^{7}$ & $\bar{p}$ \\
\hline Cycle time & 1.9 & 1 & Minutes \\
\hline Acceptances $\mathrm{h} / \mathrm{v}$ at $3.5 \mathrm{GeV} / \mathrm{c}$ & $180 \pi / 200 \pi$ & $200 \pi / 200 \pi$ & \\
\hline Emittances ( $87 \%$ beam) & & & \\
\hline $\begin{array}{l}\mathcal{E}_{\boldsymbol{h}} \quad \boldsymbol{\varepsilon}_{V} \\
\Delta \mathbf{p} / \mathbf{p} / \text { decunched } \\
/ \Delta \mathbf{p} / \mathbf{p} / \text { bunched }\end{array}$ & $\begin{array}{l}1 \pi / 2 \pi \\
1 \times 10^{4} / 2 \times 10^{-3}\end{array}$ & $\begin{array}{l}1 \pi / 1 \pi \\
1 \times 10^{4} / 1 \times 10^{-3}\end{array}$ & $\mathrm{~mm} . \mathrm{mrad}$ \\
\hline minimum extracted bunch length & $230-390$ & $200-500$ & ns \\
\hline
\end{tabular}




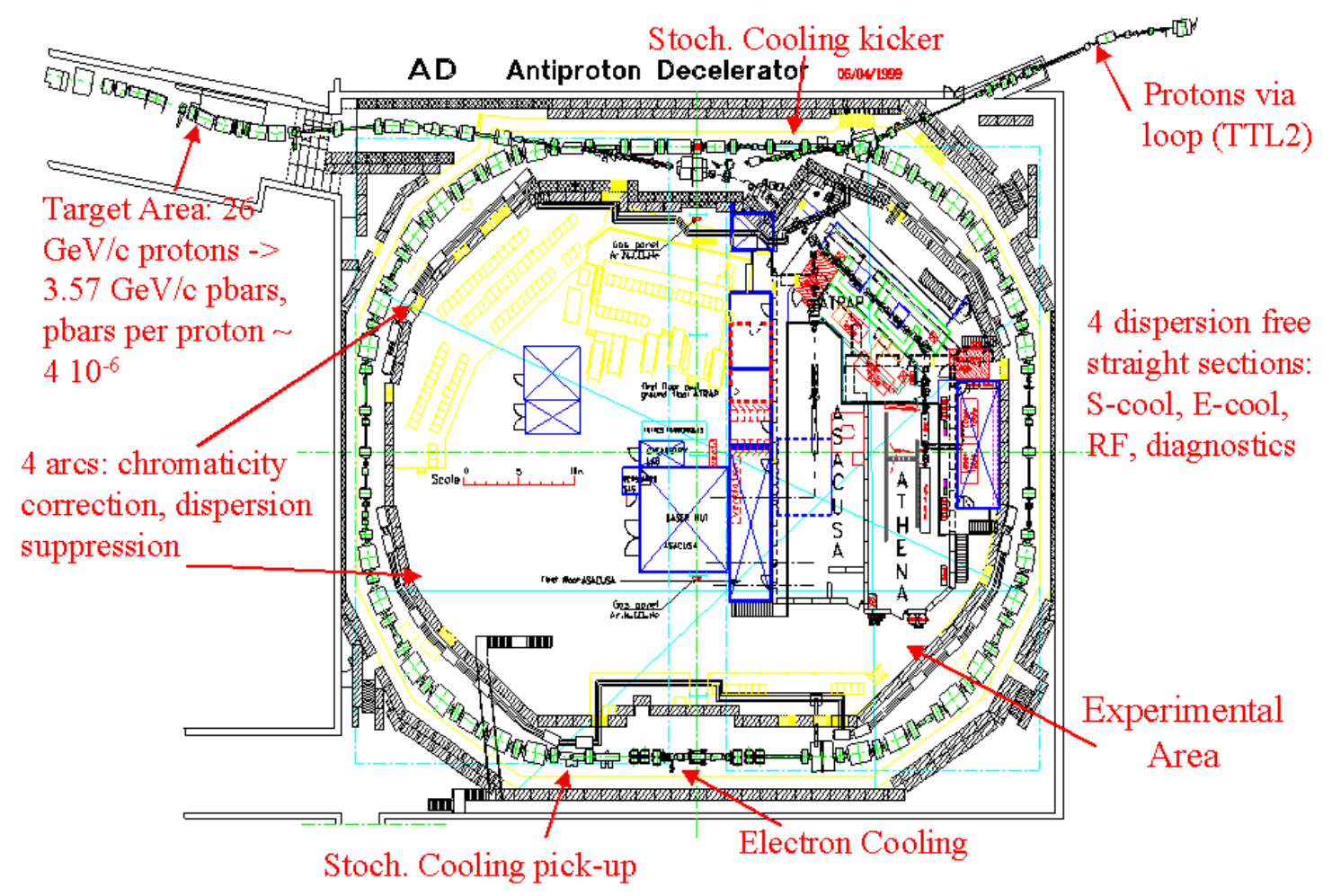

machine could be built with an investment of only 8 MCHF, necessary mainly for the upgrade and refurbishing of: vacuum, power supplies, electron cooling, rf, diagnostics, controls, and experimental areas. Compared to the scheme used until 1996 which needed four machines (the AC, AA, PS and LEAR) to provide lowenergy antiproton beams, the $\mathrm{AD}$ has brought considerable savings in operational cost (e.g. power consumption of only 0.5 instead of $8 \mathrm{MW}$ ) and manpower.

\section{CRITICAL COMPONENTS}

\subsection{Vacuum}

A vacuum pressure smaller $3 \times 10^{-10}$ torr $\left(\mathrm{N}_{2}\right.$ equiv.) is mandatory to reach the equilibrium emittance of $1 \pi \mathrm{mm}$ mrad with electron cooling at $100 \mathrm{MeV} / \mathrm{c}$ due to multiple scattering on the residual gas. High temperature baking of the machine is therefore required. Some equipment from the former AC can only stand a very gentle bake-out with a very slow rate of temperature change. Thus every bakeout takes of the order of two weeks.

During commissioning, a pressure close to the specified few $10^{-10}$ torr was reached before a leak on the tank of an extraction kicker occurred in May 2000. A definitive repair required an opening of the chamber. Therefore the leak was provisionally collimated "in situ" and repaired only during the long (December to April) shutdown
2000/2001. As a result, a local pressure bump in the year 2000 increased the scattering of the circulating antiprotons by at least a factor two, thus delaying the commissioning and early operation. Although this difficulty is now solved, it highlights the vulnerability and the inconvenience of the long delay necessary for each bake-out of the vacuum.

\subsection{Power converters}

The converters, both for the bending magnets and for the quadrupoles, form complicated networks with interlaced main and trimming supplies. The unusually high stability required to handle the cooled beam (few $10^{-4}$ at $0.1 \mathrm{GeV} / \mathrm{c}$ where the current is only $1 / 35$ of the maximum ) is an additional challenge. In the commissioning and early operation of the $\mathrm{AD}$, frequent intervention of experts was required to debug and maintain the performance of this system. These difficulties were amplified by imperfections of the control system. Nevertheless, long periods of stable operation have now become possible.

\subsection{Magnets}

All magnetic elements of the former AC were designed for DC powering, but are now cycled at a rate up to one $\mathrm{AD}$ cycle every 110 seconds. During commissioning, several quadrupoles developed water leaks. This was 
traced to small movement of the coils during the machine cycle resulting in fatigue of the copper conductors connecting different sections of the coil. The water leaks could be provisionally sealed "in situ" until a permanent solution was implemented during the winter shutdown: the coils in 28 large aperture quadrupoles were fixed more solidly by inserting spacers between the coil packages, and by re-fixing the shims. This has finally made the magnets "cycle resistant".

\subsection{Cooling systems}

The performance of the cooling systems is summarised in table 2. Whereas stochastic cooling at 3.5 and $2 \mathrm{GeV} / \mathrm{c}$ [4] works according to design expectation, the e-cooling takes a longer time than anticipated before the equilibrium emittances are obtained. To reach shorter cooling times, several measures were envisaged. The overlap of the electrons with the antiproton beam is carefully optimised and drifts in the energy are reduced by elaborate stabilisation, including feedback of the electron energy, which is not operational yet. A very high stability in the orbit and the energy of the antiproton beam are equally important. The main power supplies must be stable to a few $10^{4}$ at $100 \mathrm{MeV} / \mathrm{c}$.

The cooling performance depends on the optics of the ring. Special settings, different from the "high energy optics", are foreseen for the electron cooling plateaux.

Improvements made during the long shutdown include: re-alignment of the cooler, improvement of the magnetic circuit of the correction dipoles that align the antiproton orbit in the cooler, installation of more powerful supplies for these dipoles, and installation of two independent supplies for the correction solenoids that compensate the coupling due to the main solenoid.

The combination of all these measures has made it possible to routinely obtain the small design emittances, but the cooling-down time is still considerably longer than the design expectation.

\subsection{Beam Diagnostics}

New diagnostic devices [5] have been developed to monitor the beam characteristics like intensity, size and position with as little as $10^{7}$ pbars. At $100 \mathrm{MeV} / \mathrm{c}$ this corresponds to a circulating current of only $260 \mathrm{nA}$ which cannot be resolved by a "normal" beam current transformer nor by "straight-forward" Schottky noise diagnostics. In the beginning, commissioning was therefore done with beams of about $10^{9}$ test protons injected through the former AA-ejection line. This test proton beam is very useful for debugging but, as it circulates in the "wrong" direction, neither the cooling systems nor the directional Schottky pick-ups are usable. A delicate setting-up of the tiny $(\varepsilon=1 \pi \mathrm{mm}$ mrad $)$ test beam from the PS is required for deceleration tests. Much of the commissioning was therefore done directly with antiprotons. After elaborate shielding of the very sensitive head amplifiers against noise (cross talk) from the rfsystem, the novel closed orbit system made it possible to survey and correct the closed orbit of $\sim 10^{7}$ antiprotons, even at $100 \mathrm{MeV} / \mathrm{c}$.

The new, extremely-low-noise Schottky system was used to measure the longitudinal (sum-) current. It serves also as monitor for the momentum width and provides a good signal, especially when the beam is well cooled.

The transverse Schottky monitors, foreseen to survey the betatron tune and beam size are not yet operational. Also the profile detectors based on observing beam size via the ions (and/or electrons) created by ionisation of the residual gas, need further development. Thus for the moment, antiproton beam emittances at low momentum can only be measured destructively by moving the scrapers and observing the beam loss with scintillators. For the Q-measurement, the beam transfer function technique is used, based on the observation via a transverse Schottky pick-up of the beam response to a swept sine wave applied to a transverse kicker.

This rudimentary set of emittance and tune diagnostics proved just sufficient to set up and operate the machine, but the advent of the new transverse Schottky pick-ups and the non destructive beam ionisation profile monitors is eagerly awaited. In addition, the non-destructive survey of the ejected intensity based on a new Schottky pick up is not yet operational.

It is planned to implement the "pbars stacking mode" to accumulate antiprotons from several PS-cycles with a gain in intensity of a factor of around three. This will ease the diagnostics and also serve some users, although the stacking process is time-consuming and the flux will not increase significantly.

Table 2: Cooling Performance at the AD

\begin{tabular}{|c|c|c|c|c|c|}
\hline $\begin{array}{l}\text { Momentum } \\
{[\mathrm{GeV} / \mathrm{c}]}\end{array}$ & cooling system & $\begin{array}{l}\text { final emittances } \\
\varepsilon_{\mathrm{h}}, \quad \varepsilon_{\mathrm{v}}, \\
{[\pi \mathrm{mm} \mathrm{mr}]} \\
\text { obtained }\end{array}$ & $\begin{array}{l}(87 \% \text { beam }) \\
\Delta \mathrm{p} / \mathrm{p}_{\text {/deb }} \\
{[\%]} \\
\text { design }\end{array}$ & $\begin{array}{r}\text { Total coc } \\
\mathrm{t} \\
{[\mathrm{sec}} \\
\text { obtained }\end{array}$ & ig time \\
\hline 3.5 & Stochastic & $3, \quad 4, \quad 0.07$ & $5,5, \quad 01$ & 20 & 20 \\
\hline 2.0 & Stochastic & $3,3.5,0.03$ & $5,5, \quad 0.03$ & 15 & 15 \\
\hline 0.3 & electron & $5, \quad 2, \quad 0.05$ & $2, \quad 2, \quad 0.1$ & 16 & 6 \\
\hline 0.1 & electron & $1,2, \quad 0.01$ & $1,1, \quad 0.01$ & 10 & 1 \\
\hline
\end{tabular}




\subsection{Controls and operation}

The control system was a great help during the commissioning phase, but it is still at an early stage of evolution towards the high degree of automation [1] required for routine operation. Although the 'philosophy' is well established, a lot of application programs remain to be developed. An example is a powerful fault diagnostics programme, acquiring, storing and displaying the key parameters on all the plateaux of the cycle.

At the moment, operation is done from the local $\mathrm{AD}$ control room (ACR). A machine supervisor is responsible for the operation on a weekly basis. He is assisted by an "operations technician" who works $8 \mathrm{~h}$ shifts. The AD is scheduled to run for $3000 \mathrm{~h} /$ year from April to October and without interruption from Monday 08.00 to Friday 23.00. It is foreseen to transfer the routine operation from the local to the main PS control room later.

\subsection{Commissioning and operations challenges}

Transformation of the AC and commissioning of AD took about two years (half a year longer than originally estimated in 1996). It required considerable and permanent efforts of about 10 machine engineers/physicists and much part-time help from virtually all groups in the PS Division and many other CERN services. Points which proved more difficult than anticipated are related to the use of the "old" hardware for the new requirements, and to the handling of the low intensity beam. These are:

- The magnetic system: work had to be done on the quadrupoles to cure coil and shim movements and their related water leaks and orbit drifts. Field lag due to eddy currents had to be taken into account in the cycle program (this explains in part the lengthening of the cycle). Different saturation characteristics for special quadrupoles required additional trim supplies.

- Electron cooling system: the cooling-down time is longer than anticipated. The reasons are not fully understood. In addition, adjustment of the cooling systems requires care. The special dipoles for orbit alignment had to be improved. Compensation of the coupling proved delicate, and a separate supply was installed for the compensation solenoids (originally powered in series with the main solenoid). More work is needed for reducing the drift in energy by including the feedback of the electron energy and controlling the neutralisation of the electron beam to avoid fluctuations of the space-charge forces at higher electron currents.

- Timing and cycle editor system: this is operational but due to the complexity of the AD-cycle, it required (and still requires) frequent intervention of a controls specialist. Further development is necessary to make it more robust and user-friendly.

- Vacuum system: The design pressure has been obtained, but commissioning was plagued by leaks which demanded a very long bake-out for a definitive repair.

- Diagnostics: Absence of non-destructive emittance and ejected intensity measurements make the operation somewhat difficult. The Q-measurement, for the moment, leads to a beam blow-up. The interference of residual noise from the rf-system with the longitudinal Schottky signals and the closed orbit measurements has been eliminated, and the beam size measurement with scrapers is operational. Thus it is possible, although time consuming, to set up the machine entirely with the antiproton beam.

There were also some good surprises during commissioning, including:

- $\quad$ After setting up, the intensity $2 \times 10^{7}$ at $0.1 \mathrm{GeV} / \mathrm{c}$ is routinely reached; this factor two gain is due to a high deceleration efficiency and to an improvement of the production beam from the PS.

- The complicated optics of the AD lattice work very satisfactorily. Response matrix measurements and orbit collection proved extremely useful, identifying deviations and suggesting corrections.

- Stochastic cooling at 3.5 and $2 \mathrm{GeV} / \mathrm{c}$ was quickly achieved and exceeds the design performance.

- The ultra-low noise orbit system could be improved to $+/-0.2 \mathrm{~mm}$ precision for $2 \times 10^{7}$ antiprotons at $0.1 \mathrm{GeV} / \mathrm{c}$.

- The new longitudinal Schottky pick-up and its new (DSP-based) acquisition system allows the weak antiproton intensity to be monitored. On the basis of the diagnostics developed (measurements of intensity, orbits, tunes, emittances, coupling, response matrices), a much larger part of the commissioning could be performed with antiprotons than originally anticipated.

\section{FUTURE DEVELOPMENTS}

To consolidate the design performance, further work is necessary. A special effort is needed to obtain high stability and small emittances of the extracted beam consistently. Stacking at injection will be implemented if the higher intensity per pulse is required for special physics needs. To make this useful, an active feedback system to counteract the coherent instability will probably have to be put into operation.

As another option, "multiple extraction" could be envisaged, e.g. by capturing the $100 \mathrm{MeV} / \mathrm{c}$ beam into several buckets which are kicked out individually. Three bunches can be provided relatively easily with the rfsystem that works at harmonic 3 from 300 to $100 \mathrm{MeV} / \mathrm{c}$. The time between ejections is constrained by the repetition time of the kicker on one hand and by the beam lifetime on the other.

The options mentioned so far are more or less straight forward extrapolations from the design performance. Developments beyond this would be limited by machine 
physics issues but probably even more so by the space available in the Hall and by scheduling difficulties. This applies to the request for more experiments and/or more exotic extracted beam patterns.

\section{CONCLUSION}

The conversion of the AC ring to a Decelerator slowing down antiprotons from 3.5 to $0.1 \mathrm{GeV} / \mathrm{c}$ required a long and tedious commissioning period. Regular operation with good beam characteristics started in July 2000 and by now (June 2001) the performance is close to design expectations. Work is still needed to further improve and consolidate various systems and to ease the operation.

\section{ACKNOWLEDGEMENTS}

The AD Project would not have been possible without the help of many groups at CERN and especially the AD user community which provided strong financial and manpower support. Special thanks are due to all the members of the AD commissioning team for their heroic efforts.

\section{REFERENCES}

[1] S. Baird et al, "Design Study of the Antiproton Decelerator," AD, CERN/PS 96-43 (AR).

[2] A.M. Lombardi, W. Pirkl, Y. Bylinsky, "First Operating Experience with the CERN Decelerating RFQ for Antiprotons", this Conference.

[3] P. Beloshitsky et al, "Optics of the Antiproton Decelerator," this Conference.

[4] C. Carli and F. Caspers, "Stochastic Cooling at the CERN Antiproton Decelerator, CERN/PS 2000-024 (AE), EPAC 2000, Vienna, Austria.

[5] V. Chohan et al, "Beam Measurement Systems for the CERN Antiproton Deceleration (AD)," this Conference.

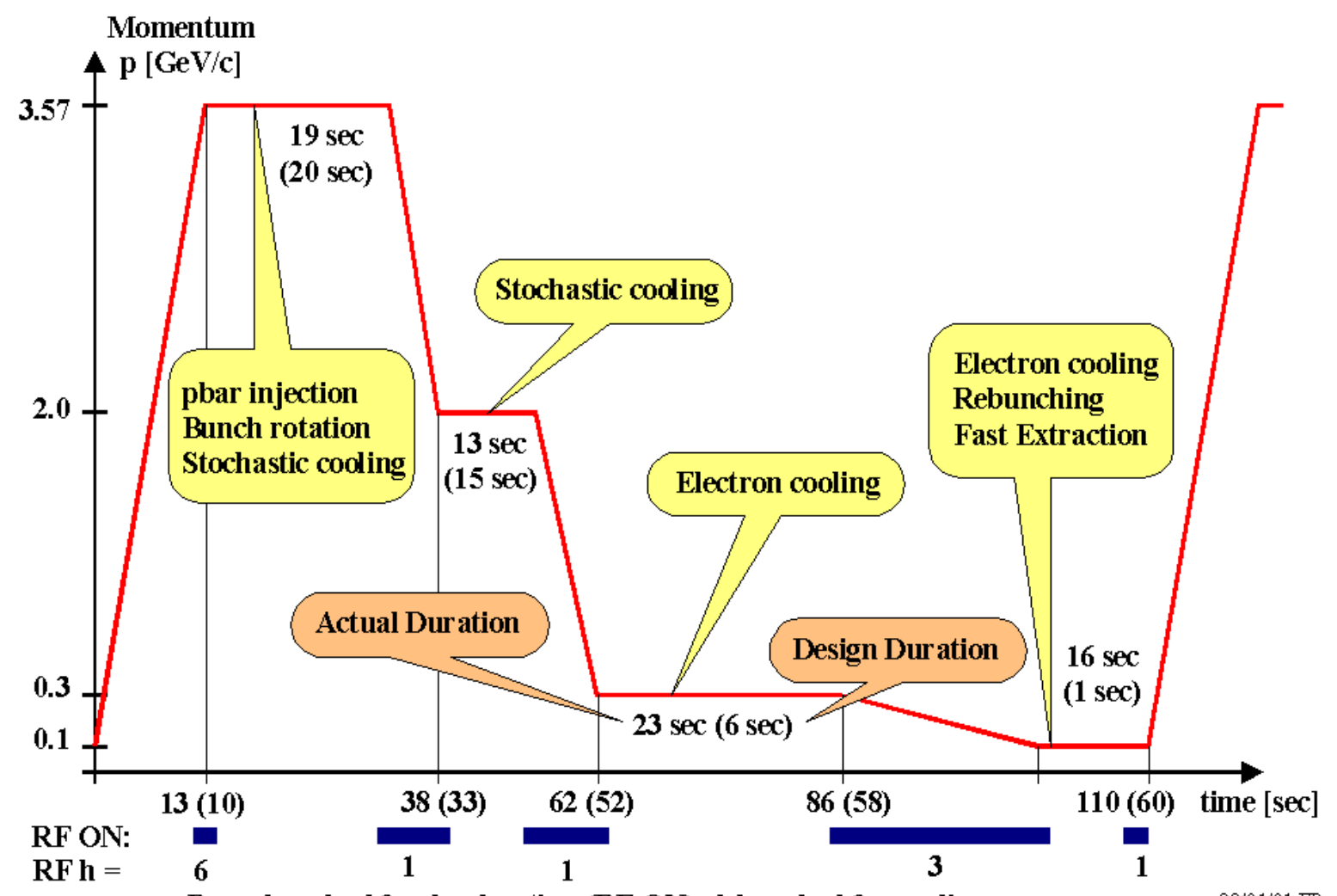

Beam bunched for deceleration (RF ON), debunched for cooling

22/01/01 FP

Figure 2: AD Deceleration Cycle 